\title{
AIAA-2001-0758 \\ Cryogenic Balance Technology at the \\ National Transonic Facility (Invited)
}

P.A. Parker

NASA Langley Research Center

Hampton, Virginia

39th AIAA Aerospace Sciences Meeting \& Exhibit

$$
\begin{gathered}
\text { 8-11 January } 2001 \\
\text { Reno, Nevada }
\end{gathered}
$$



AIAA-2001-0758

\title{
CRYOGENIC BALANCE TECHNOLOGY AT THE NATIONAL TRANSONIC FACILITY
}

\author{
P.A. Parker ${ }^{*}$ \\ NASA Langley Research Center \\ Hampton, Virginia 23681
}

\begin{abstract}
This paper provides an overview of force measurement at the National Transonic Facility (NTF). The NTF has unique force measurement requirements that dictate an integration of all aspects of balance design, production, and calibration. An overview of current force measurement capabilities is provided along with new balance development efforts. Research activities in the areas of thermal compensation and balance calibration are presented. Also, areas of future research are detailed.
\end{abstract}

\section{Introduction}

The National Transonic Facility (NTF) is a fan driven, closed circuit, continuous flow pressurized wind tunnel. It operates in a temperature range between +150 degrees Fahrenheit to -260 degrees Fahrenheit and a pressure range from atmospheric up to 9 atmospheres. It has a 2.5 meter square test section. This cryogenic wind tunnel utilizes cold gas as the test medium, which increases Reynolds number by reducing the kinematic viscosity and increasing the density of the gaseous test medium. The NTF is a unique national laboratory for the investigation of Reynolds number scaling effects. The research data obtained from the NTF is used by commercial industry and the Department of Defense for the purpose of research and development programs, validation of scaling theory, and the validation of computational fluid dynamics codes. ${ }^{1}$

Direct force and moment measurement of aerodynamic loads is fundamental in wind tunnel testing at the NTF. These measurements are provided by an instrument known as a force balance. Force balances remain as the state-of-the-art instrument used for high precision

"Research Scientisı, Model Systems Branch, Member AIAA

Copyright 2001 by the American Institute of Aeronautics and Astronautics, Inc. No copyright is asserted in the United States under Title 17, U.S. Code. The U. S. Government has a royalty-free license to exercise all rights under the copyright claimed herein for Governmental Purposes. All other rights are reserved by the copyright owner. aerodynamic force measurements in wind tunnel testing. Since the 1940s, force balance design has been based on structural flexural elements outfitted with strain sensors. The testing requirements of the NTF push the limits of this force measurement technology. Their structural design is challenging and the measurement accuracy requirements are demanding.

Development of cryogenic balances at NASA Langley Research Center (LaRC) began in the 1980s. At that time, there were no other cryogenic facilities in the world and many basic procedures needed to be developed. $^{2}$ These developments enabled the construction of the first NTF balance in 1982 . Throughout the years, there have been many improvements in the areas of balance structural design, fabrication techniques, strain sensors, data acquisition systems and calibration loading systems. At the NTF, there are two main types of force balances, internal and semi-span. Internal balances are used for full-span model testing and semi-span balances are used for semi-span, or half-model, testing.

Due to the unique force measurement requirements at the NTF, the balance engincer must be engaged in tunnel operations. The balance engineer must realize that the balance is an integral part of the testing and measurement system. The balance cannot be viewed as an isolated instrument. Its performance and reliability are major factors in the research data quality and productivity of the NTF. Delays due to balance malfunction can be costly and time consuming.

In recent years, NTF force measurement has focused on two main areas, maintenance of operational balances and advancement of NTF balance technology. First, to develop documented procedures that provide a high level of quality control in the production and maintenance of the current operational NTF balances. These procedures start with the balance fabrication and continue through periodic in-service inspections and diagnostics. Second, to advance the state-of-the-art in balance design and calibration. In specific, the goals 
are to increase the accuracy, load range, and improve the dynamic characteristics of the NTF balances.

This paper will provide an overview of current NTF balance capabilities and areas of research and development. A review of operational balance capabilities is discussed followed by near-term new capabilities. The areas of rescarch and development include thermal compensation and balance calibration techniques. The author acknowledges that are other centers of cryogenic balance development in the world, but this paper will focus primarily on work performed at NASA LaRC.

\section{General Description of NTF balances}

This section is intended to serve as a brief overview of force balance fundamentals. A detailed description of force balance design concepts is provided in Reference 3. A typical internal strain-gage balance is shown in Figure 1.

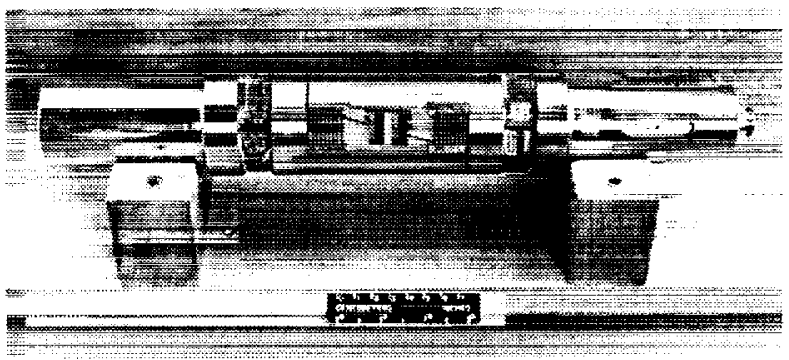

Figure 1. Internal six component NTF force balance (NTF-113B).

The force balance is a complex structural spring element designed to deflect a specified amount under applied load. The balance is mounted internally to the model, and the components measured by the force balance consist of normal (NF), axial (AF), pitching moment (PM), rolling moment (RM), yawing moment (YM), and side force (SF), see Figure 2.

This deflection of the balance under load results in a change in strain in the flexural clements. This differential strain is measured with six Wheatstone bridges, each consisting of four foil resistive strain gages. Each bridge is designed to primarily respond to the application of one of the six components of load. The electrical response of these bridges is proportional to the applied load on the balance. This strain, as a function of load, forms the basic concept of force balance measurements.

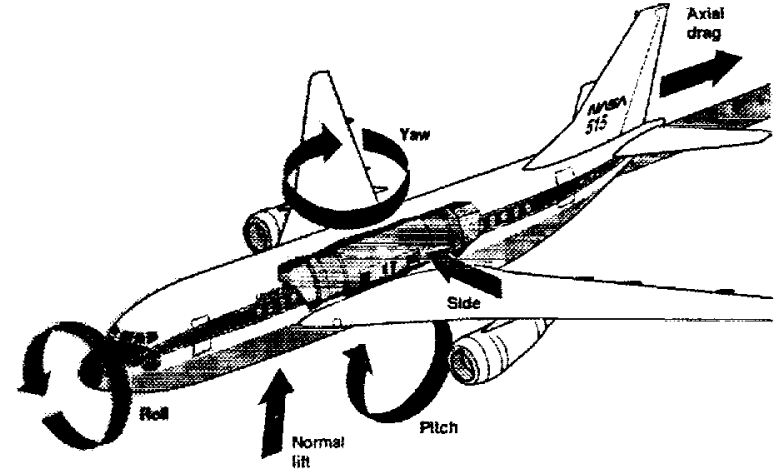

Figure 2. Measured aerodynamic forces and moments.

The balance flexural elements are optimized such that the magnitude of the strain response is approximately the same for the individual application of the full-scale load of each component. The magnitude of the components is not the same, and therefore the flexural elements do not have the same spring constant in all axes. Typical balance load ratios of lift to drag are 16 to 1 . This dictates that the force balance must be carefully designed in order to achieve an accurate measurement of drag in the presence of a large lift force.

Ideally, each balance response signal would respond to its respective component of load, and it would have no response to other components of load. This is not entirely possible. The response of a particular balance channel to the application of other components of load is referred to as an interaction effect. Balance designs are optimized to minimize these interaction effects. Ultimately, a mathematical model must correct these unwanted interaction effects. A calibration experiment provides the data to derive an adequate mathematical model. This model is referred to as the calibration coefficient matrix.

The force balance has many unique requirements as compared to other measurement devices. The balance is a high-precision measurement instrument as well as a critical structural component of the model support system. Balance design requires an optimization of the measurement sensitivity and the structural safety margins. To achieve high sensitivity the strain gage flexures should produce maximum allowable strain under applied aerodynamic loading. The allowable strain is determined by the balance material properties and the required margin of safety. Furthermore, this optimization must be performed in six degrees of freedom, achieving equal performance for all measured components of aerodynamic load. Fulfilling this dual 
requirement is one of the most challenging areas of force balance design. The balance engineer must maintain the viewpoint that the first priority is to achieve high-accuracy measurements of the forces and moments exerted on the model during acrodynamic testing. The accurate resolution of forces and moments is considered paramount in all decisions regarding balance production. Any decision made that deteriorates the measurement accuracy must be scrutinized to determine its necessity.

\section{Current NTF Balance Capabilities}

All NTF balances are constructed from a single piece of high strength maraging steel to produce a transducer quality spring. The monolithic construction ensures minimum mechanical hysteresis within the balance. The material selected provides acceptable spring qualities and maintains acceptable toughness at cryogenic temperatures for this critically stressed component. The model attachment, or metric end, of most NTF internal balances consist of a precisionground cylinder secured to the model with an interference fit dowel pin to the model. On the sting side, or non-metric end, the internal balances provide a cylindrical taper fit with a key and set screw flats for securing and removing from the sting. Semi-span balances, which are mounted in the tunnel wall, utilize a flange type attachment.

The nominal diameter for internal balances versus the maximum NF capacity is depicted in Figure 3. Note that an approximate diameter is used for the NTF- 116 , it has an octagonal cross section A detailed list of all NTF balances, including their full-scale load limits on all six components of load is given in Reference 4.

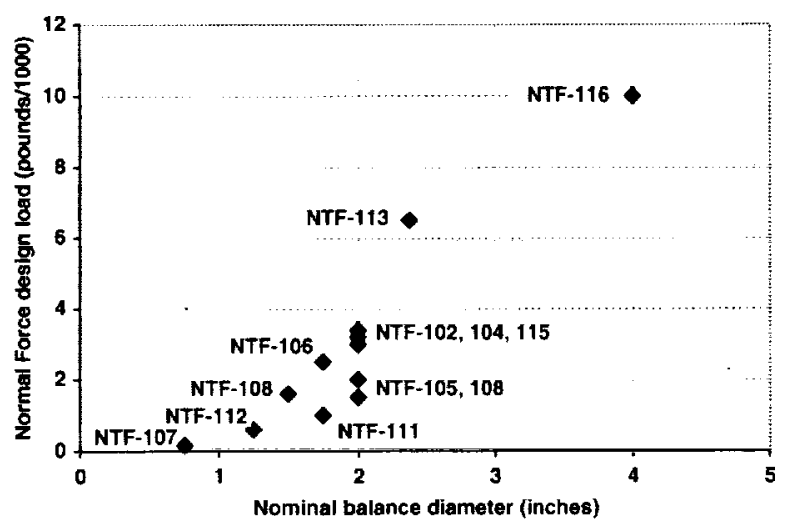

Figure 3. Normal force load capacity versus the nominal balance diameter.

\section{Internal Balance Instrumentation}

The NTF-113 series of balances are the most commonly used due to their high load range. Most of the balances in this series utilize the latest gage installation techniques and are instrumented with 48 individual strain-gages that provide twelve Wheatstone bridge outputs. This provides two bridges per component. The two sets of six bridges are electrically isolated from each other and are powered by independent excitation sources. Reliability is the main reason for providing the redundant set of bridges. Since testing in a cryogenic facility is very costly, and the possibility of replacing a balance during a tunnel test severely impacts tunnel productivity. An added benefit of the redundant bridge approach is improved troubleshooting time. Typically, if both bridges on the balance express the same behavior, the phenomena is load related in contrast to an electrical problem.

Four of the six components of load are calculated by combining certain bridges using the equations as below.

$$
\begin{aligned}
& N F=N 1+N 2 \\
& P M=N 1-N 2 \\
& Y M=S 1-S 2 \\
& S F=S 1+S 2
\end{aligned}
$$

where,

NF, PM, YM, SF are the component outputs, and $\mathrm{N} 1, \mathrm{~N} 2, \mathrm{~S} 1, \mathrm{~S} 2$ are individual bridge signals

This type of balance is commonly referred to as a moment balance. This configuration is utilized to physically isolate an entire strain-gage bridge to one longitudinal position. Also, the strain is measured utilizing a Poisson ratio gage layout, ${ }^{5}$ orienting a primary gage perpendicular to a Poisson ratio gage. The two gages are wired in a half bridge configuration and their active grids are in close proximity to each other. By strategic positioning of the strain gage bridges, temperature differences within a half bridge are practically eliminated. This gage orientation is utilized for all bridges except for axial force and roll moment. A disadvantage of this configuration is a reduced sensitivity of the strain measurement.

The strain gage type and preparation are unique to NTF balances. The gage type is selected to minimize the change in sensitivity of the balance as a function of temperature, it is referred to as a modulus compensated gage strategy. The change in sensitivity is typically on 
the order of one percent of full-scale output. A temperature based sensitivity shift coefficient is derived by performing calibration at cryogenic temperature, providing a corrective means for this residual effect. This calibration procedure is discussed in more detail later in this paper.

A unique procedure performed by LaRC, is the process of gage matching. ${ }^{6}$ The gage matching procedure allows for individual gage characteristics to be determined before the gages are installed on the balance. It also provides a means to select the optimum combination of four strain gages in each bridge. This process involves the installation of 16 strain gages onto a test fixture, that is fabricated from a low coefficient of thermal expansion material to isolate the gage performance from the base material. The test fixture is then subjected to a cryogenic temperature excursion. Data is recorded using high-precision strain gage quarter bridge completion units. The response from all 16 strain gages is recorded, as well as the temperature of the test fixture. An optimization algorithm sorts through the 16 strain gages configuring them in all possible four-gage combinations. The four-gage bridges are then sorted according to performance metrics. These metrics include the magnitude of the bridge response as a function of temperature and the linearity of the response. Typically, three usable bridges can be obtained from one group of 16 gages. Once matching is complete, the strain gages are removed from the test fixture and filed into a series of trays until they are ready to be installed on the balance. This procedure is repeated in groups of 16 gages at a time until enough bridges have been matched for the particular balance to be gaged. Further selection is then made to choose the best of the possible bridges to be used for the axial force component. Gage matching is a time consuming and labor intensive process, but its merits have been demonstrated in the final thermal response characteristics of the balance.

Testing at cryogenic temperatures requires a constant monitoring of the balance average temperature and temperature gradients. Nine temperature measurements in the form of Platinum Resistance Temperature Detectors (PRTD) are available for monitoring the balance thermal condition. Three of the PRTDs provide a temperature at the forward (model end), middle and aft end (sting end) of the balance. Six of the sensors are located in strategic locations near the axial measuring section to perform thermal gradient compensation.

The NTF environment requires that the balance electrical connections be protected from moisture.
Moisture causes corrosion and electrical shunting on exposed electrical connections. The installation of any material on or near the strain gage can cause undesirable responses due to temperature. This is primarily due to differential thermal contraction between the strain gage, the base material, and the electrical wiring. Therefore, the goal is to provide a moisture resistant barrier that has a minimal influence on the performance of the balance. The development of moisture resistant coatings for NTF balances, using various materials and techniques, has required many years of research and testing. Various methods have been utilized over the years, each with there own drawbacks in terms of performance as a moisture barrier, influence on the balance measurements, and reliability. The result of this research has determined that the skill of the gage technician, the installation procedure, and the selection of the coating material have equally significant contributions to the success of the coating.

The current moisture resistant coating procedure integrates all of these aspects. First, the purity of the coating material and the cleanliness of the balance are critical to success. The balance goes through a series of cleaning procedures that removes foreign material and eliminates residue from the cleaning agents. The material used for the coating is a solvent-thinned form of nitrile rubber. This material is applied in a lowhumidity, low-temperature chamber, see Figure 4 . The low-humidity environment helps to ensure that no moisture is trapped under the coating material. The low-temperature aspect slows the curing process of the coating allowing it to flow around the electrical connections while minimizing the production of air pockets within the coating. The coating material is automatically dispensed with a fine syringe-like nozzle under a microscope. After the material has been applied to the balance, a pressurized curing procedure is utilized. The balance is placed in a pressure vessel that is purged with nitrogen gas and then pressurized for 10 15 hours. The pressure cure further enhances the sealing process and continues the minimization of air pockets within the material. Time constraints on the curing of the coating material allow for the simultaneous coating of only a few bridges at one time. The same process is repeated until all of the exposed electrical connections on the balance have been coated. 


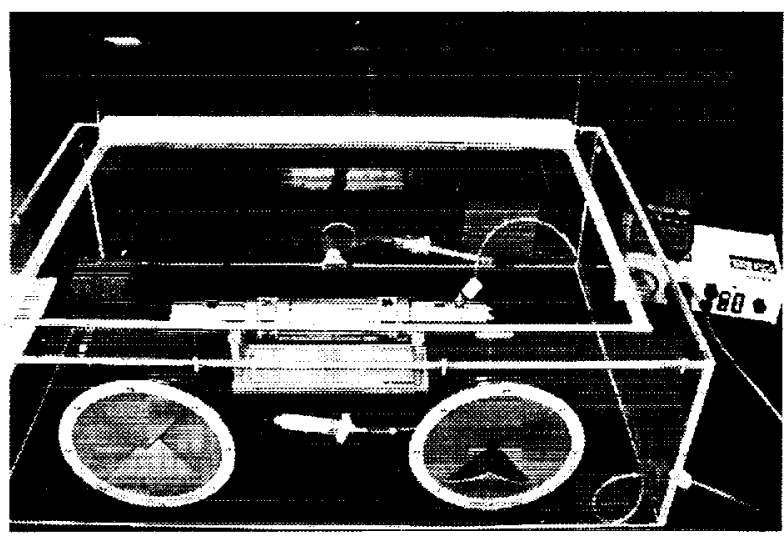

Figure 4. Laboratory chamber used for application of moisture resistant coating.

Once the balance is completely instrumented, thermal compensation begins. This phase involves constant-load thermal excursions to characterize and compensate for electrical response of the balance as a function of temperature. The first step in this phase is to ensure that the moisture resistant coating has not influenced the balance thermal response. Data collected from thermal excursions prior to the installation of the moisture resistant coating is compared to the thermal excursions after coating. If there are significant effects, the coating is removed and re-installed. This iterative process continues until the coating effects are negligible. Thermal response due to the moisture resistant coating could be compensated for by electrical or mathematical techniques, but LaRC has found the performance over time of the coating to be unreliable and therefore the compensation could become inaccurate. It is better to minimize, or ideally eliminate the effects of the coating rather than compensate for these effects. Once the coating is determined to be satisfactory, the response of the strain gages as a function of temperature can be performed. This procedure includes compensation for isothermal balance conditions and for thermal gradient conditions. Figure 5 shows the cryogenic chamber used in the laboratory for thermal testing. The details of thermal compensation are discussed in detail later in this paper.

It should be noted that the successful execution of the above procedure of instrumentation installation requires an experienced and talented gage technician. The quality of the balance performance and its long-term stability is directly related to the careful attention to detail during the gage installation process. The gage work on the NTF balances has evolved into an art, not a science.

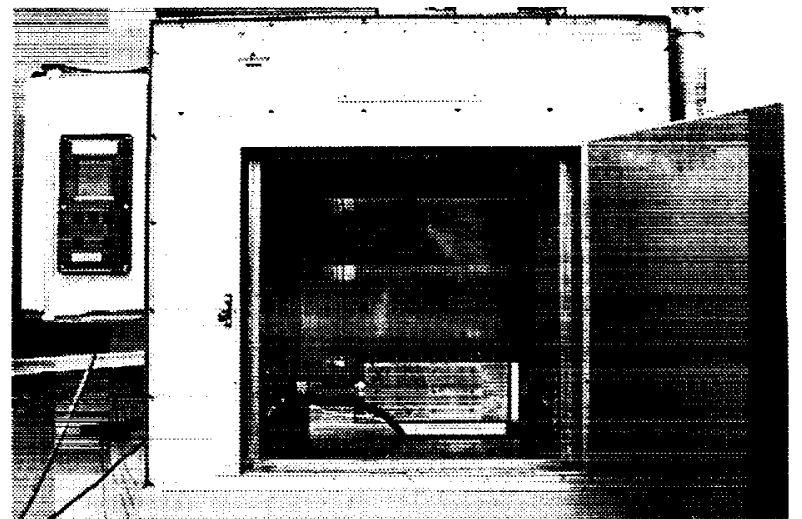

Figure 5. Environmental chamber used in laboratory thermal testing.

\section{Internal Balance Calibration}

Current NTF balances undergo an extensive sequence of calibration. Force balance calibration is the most critical phase in the balance production. It serves as the final test of the balance performance, and quantifies the measurement accuracy. A detailed description of the balance calibration procedure at LaRC is provided in Reference 7. There is a significant, focused effort at LaRC on improving the calibration process in the areas of accuracy, productivity, and cost. LaRCs research and development efforts in these areas are presented later in this paper.

A typical NTF internal balance calibration procedure is partitioned into three main areas: application of loads with the balance at room temperature, application of loads with the balance at -290 degrees Fahrenheit, and the determination of balance deflection constants. Initially, the balance is loaded at room temperature following a load sequence that applies one load at a time up to six loads simultaneously. This sequence includes approximately 800 load points and takes three to four weeks to complete based on the load range. The data collected from this process is used to derive a quadratic mathematical model. The quality of fit of this model is determined by calculating the residual errors of data used to derive the calibration matrix as well as a series of proof load sequences. The accuracy of the calibration is defined by the mean residual error plus and minus two times the standard deviation of the errors. This provides a $95 \%$ confidence interval of the quality of fit of the calibration model. Typical accuracy is provided in Table I for the NTF-113B balance.

A calibration of the balance at cryogenic temperatures follows the room temperature calibration. The purpose of this calibration is to determine the correction 
coefficients for the change in bridge sensitivity as a function of temperature. The load combinations performed at cryogenic temperatures are compared to the room temperature results in a relative manner.

Table I. Typical measurement accuracy of a NTF internal balance

\begin{tabular}{|l|c|}
\hline \multicolumn{1}{|c|}{ Component } & $\begin{array}{c}\text { Accuracy, 2 } \mathbf{~} \\
\text { (percent of full-scale load) }\end{array}$ \\
\hline Normal Force & $0.11 \%$ \\
\hline Axial Force & $0.19 \%$ \\
\hline Pitching Moment & $0.11 \%$ \\
\hline Rolling Moment & $0.19 \%$ \\
\hline Yawing Moment & $0.18 \%$ \\
\hline Side Force & $0.18 \%$ \\
\hline
\end{tabular}

Primary balance deflection constants are also determined in the laboratory. These constants provide the rotation about the balance moment center in degrees as a function of each individually applied load. The deflection constants are used in the determination of aeroelastic divergence limits at the wind tunnel.

\section{Periodic Internal Balance Inspection}

A periodic inspection procedure has been recently instituted. The goal of this inspection sequence is to maintain high quality force measurements and increase balance reliability by tracking the condition of the balance over time. The periodic inspections include a comprchensive visual microscopic inspection of the strain gages, temperature sensors, wiring, and moistureresistant coating. Digital photographs of specific areas of concern follow this microscopic inspection. Through this chronicling of the balance condition, before and after each wind tunnel test, a determination can be made as to whether repair or refurbishment is required prior to the next wind tunnel entry. The balance structure is inspected in a similar way to determine if mechanical damage and thereby repair is necessary. On an annual basis the balance undergoes a magnetic particle inspection for any indication of structural cracks. Each balance has its own logbook that details the results of the inspections and any corrective actions taken.

The balance instrumentation cable is also inspected and repaired if necessary. The cable used for the most frequently used balances is detachable, and therefore repairs can be made independent of the balance itself. Not only is the cable detachable, but it is also interchangeable with other balances. The cable is the most likely component to be damaged during the installation of the balance onto the model support system, and therefore this independent arrangement is optimum for maintenance.

The electrical response of the balance is recorded each time the balance enters or exits the balance inventory. Attention to detail regarding orientation and the data system used is important in recording this electrical reading and comparing it to previous data. It is an indication of the overall health of the balance. It also aids in isolating when and where damage, if any, could have occurred.

Operational NTF balances have proven to have adequate data quality and be very reliable. Development of new balances discussed below will provide new force measurement capabilities to the NTF.

\section{Advanced Internal Balance Design, NTE-116A}

In the area of balance design and analysis, LaRC has developed a new internal balance to meet static and dynamic design requirements. The development of this balance combined balance design experience and stateof-the-art finite element analysis tools. These finite element tools have been used to analyze an exiting balance design ${ }^{8}$, but this development effort marked the first time that finite element analysis was utilized as a balance design tool at LaRC. The new balance is designated as the NTF-116A. The NTF-116A advanced balance design provides a five to ten time increase in stiffness while maintaining the sensitivity of the current state-of-the-art NTF force balances. The primary goals of this new balance were to increase stiffness and load capacity. Furthermore, the balance was constrained to fit within an existing model.

Increased stiffness was required in order to contribute to a comprehensive effort to suppress model vibrations that occur during wind tunnel testing. Since the start of tunnel testing at the NTF in 1984, there have been problems with unexpected model vibrations. Dynamic loads associated with vibratory motion may approach the structural limits of the model or its support system, which poses the risk of model loss and facility damage and may restrict the allowable range of test variables. Unsteady forces and displacements can seriously affect data quality. Furthermore, vibrations can introduce measurement uncertainties such as bias errors in inertial devices used for measuring angle of attack and in force balance readings due to centrifugal acceleration. ${ }^{9}$ Model vibrations can also overload force balances and stings and cause the model to foul. 
The concept of model system de-tuning has been proposed as a component of the overall solution to this problem. The basic idea is that if the model system does not have the same natural frequencies as the sources of high energy forcing function excitations, then modal coalescence would not occur, and the model system would not experience unacceptable vibrations. This effectively de-tunes the model system from the wind tunnel model support system. To incorporate model system de-tuning into the design phase of a balance, it requires the a priori ability to predict the model system modes and their associated mode shapes. This prediction can be performed using finite element tools or a simplified analytical model simulator. ${ }^{10}$ The full implementation of model system de-tuning will also require the development of advanced model support systems that are also designed to meet dynamic design requirements.

In a broad sense, sting mounted model vibrations exist in virtually all wind tunnels, but can be particularly severe in the areas of high angle of attack and high pressure facilities operating at high dynamic pressures." The approach used in this balance design can be readily applied to other wind tunnel model systems.

The possibility of stiffening the force balance had been considered previously, but never pursued due to concerns regarding a loss in balance sensitivity. Even though the balance sensitivity is based on the measured strain on the flexural elements, the overall balance deflection is not dominated by the flexural rigidity of these measuring elements. Rather, cross-sectional shape and length dominate the overall balance stiffness. These parameters as well as the mechanical connections were optimized to utilize the available space within the model.

Balance NTF-116A is shown in Figure 6. The crosssection of this new balance is octagonal. The octagonal shape is not symmetric. Its width is greater than its height to provide higher stiffness in the yaw direction. This shape was chosen to fit within the upper portion of the fuselage within the model. An elliptical shape was also considered, but the added cost of fabrication was not justified based on the marginal benefits.

The mechanical connections on both ends of the balance are flanges. LaRC semi-span balances have successfully used flange fits, but the NTF-116A is the first internal LaRC balance to utilize this type of connection. Flange joints provide a stiffer mechanical connection between the balance and the model support system as well as between the balance and the model. The flange design also provided the ability to re-locate the balance within the model in order to efficiently use the available volume. A further benefit of the flange design is the ability to fabricate short adapters that will facilitate the use of the NTF-116A on existing model support system hardware. The non-metric (sting-end) flange includes a combination of a precision ground surface, bolts, dowel pins, and a shear lip to secure and position the balance. The metric end flange is similar but does not include a shear lip. Both flanges have through bolted connections. This design eliminates any threads in the balance. Threads in the balance are undesirable because they can create a non-uniform strain field near the sensitive measurement sections.

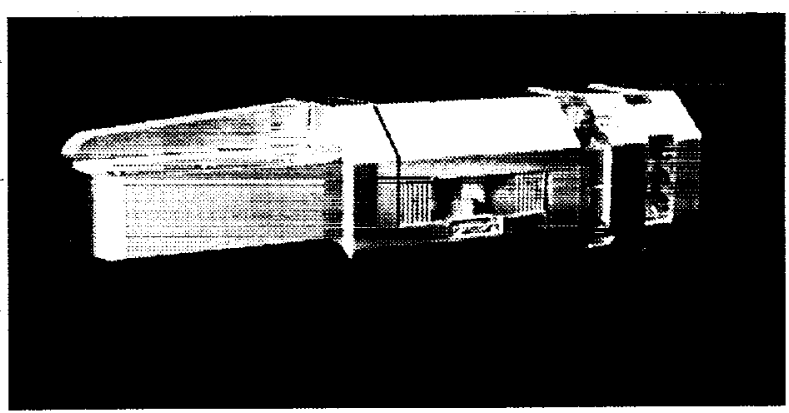

(a) Balance without shielding. Figure 6. Photograph of balance NTF-1 16A.

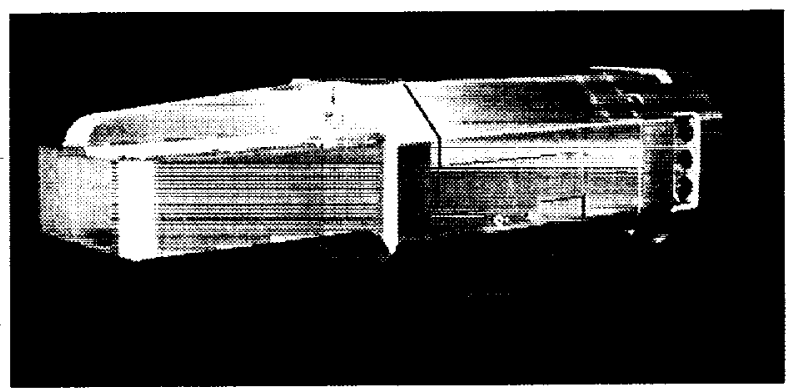

(b) Balance with cover shields installed.

Figure 6. concluded.

The balance length was shortened by reducing the number of strain-gage measuring sections to a total of two, compared to a conventional design that has three. The first measurement section is dedicated to axial force. The second section is used for the measurement of all other components. The location of this second section is aft of the axial force measurement section, and thereby reduces the number of wires that cross the sensitive axial force flexures, as compared to a conventional balance layout. The reduction in length provides a significant increase in stiffness. 
The design specifications in terms of load capacity and estimated stiffness are provided in Tables II and III. The values for the NTF-113 series of balances are provided for comparison.

Table II. Balance NTF-116A design load capacity

\begin{tabular}{|l|c|c|}
\hline Component & NTF-113 & NTF-116A \\
\hline $\begin{array}{l}\text { Normal Force } \\
\text { (pounds) }\end{array}$ & 6,500 & 10,000 \\
\hline $\begin{array}{l}\text { Axial Force } \\
\text { (pounds) }\end{array}$ & 400 & 700 \\
\hline $\begin{array}{l}\text { Pitching Moment } \\
\text { (inch-pounds) }\end{array}$ & 13,000 & 40,000 \\
\hline $\begin{array}{l}\text { Rolling Moment } \\
\text { (inch-pounds) }\end{array}$ & 9,000 & 16,000 \\
\hline $\begin{array}{l}\text { Yawing Moment } \\
\text { (inch-pounds) }\end{array}$ & 6,500 & 24,000 \\
\hline $\begin{array}{l}\text { Side Force } \\
\text { (pounds) }\end{array}$ & 4,000 & 4,000 \\
\hline
\end{tabular}

Table III. Balance NTF-116A

estimated rotational stiffness

\begin{tabular}{|l|c|c|c|}
\hline Component & $\begin{array}{c}\text { NTF-113 } \\
\text { (measured) }\end{array}$ & $\begin{array}{c}\text { NTF-116A } \\
\text { (estimated) }\end{array}$ & $\begin{array}{c}\text { Ratio } \\
\left(\mathrm{K}_{113} /\right. \\
\left.\mathrm{K}_{116}\right)\end{array}$ \\
\hline $\begin{array}{l}\text { Pitching Moment } \\
\text { (inch- } \\
\text { pounds/radian) }\end{array}$ & 955,000 & $4,100,000$ & 4.3 \\
\hline $\begin{array}{l}\text { Rolling Moment } \\
\text { (inch- } \\
\text { pounds/radian) }\end{array}$ & 365,750 & $4,300,000$ & 11.8 \\
\hline $\begin{array}{l}\text { Yawing Moment } \\
\text { (inch- } \\
\text { pounds/radian) }\end{array}$ & 859,500 & $4,900,000$ & 5.7 \\
\hline
\end{tabular}

NTF-116A features a similar suite of instrumentation included on the NTF-113 series of balance. This includes a redundant set of bridges and twelve platinum temperature sensors. The balance instrumentation is completely shielded with metallic non-contacting cover shields. These shields are intended to increase the ruggedness of the balance, but are also expected to improve the thermal stability. At the present time, the NTF-116A has been fabricated and is in the strain gage installation phase.

Wind tunnel testing of the NTF-116A will help determine the feasibility of the concept of model system de-tuning. As further research is performed at the NTF on the suppression of model system dynamics, it may be required to change the dynamic design requirements for the force balance. In fact, increasing the flexibility of the force balance has been proposed to increase the divergence pressure limit. ${ }^{12}$ Regardless of the dynamic requirements, this approach has effectively de-coupled the balance sensitivity from the overall balance stiffness. The development of the NTF-116A has demonstrated the ability to satisfy both static and specific dynamic requirements.

\section{Semi-Span Balance Development. NTF-117S}

There has been a significant effort to develop a semispan testing capability at the NTF. The development of this testing technique has had a definite impact on the balance requirements. The semi-span balance was originally fabricated, instrumented and calibrated for cryogenic operation like all other internal NTF balances. The actual operation of the balance was later determined to be at ambient temperature regardless of the tunnel test section temperature. Currently there is only one operational semi-span NTF balance, the NTF$114 \mathrm{~S}$. As a result of the semi-span test technique development, a new higher capacity balance has been fabricated and designated as the NTF-117S. Balance NTF-117S, with a 12,000 pound normal force capability, doubles the lift capacity of the NTF-114S, Table IV contains the design loads of the NTF-I17S with the NTF-114S specifications provided for comparison.

Table IV. Balance NTF-117S design load capacity

\begin{tabular}{|l|c|c|}
\hline Component & NTF-114S & NTF-117S \\
\hline $\begin{array}{l}\text { Normal Force } \\
\text { (pounds) }\end{array}$ & 6,100 & 12,000 \\
\hline $\begin{array}{l}\text { Axial Force } \\
\text { (pounds) }\end{array}$ & 1,300 & 1,800 \\
\hline $\begin{array}{l}\text { Pitching Moment } \\
\text { (inch-pounds) }\end{array}$ & 70,000 & 90,000 \\
\hline $\begin{array}{l}\text { Rolling Moment } \\
\text { (inch-pounds) }\end{array}$ & 353,800 & 670,000 \\
\hline $\begin{array}{l}\text { Yawing Moment } \\
\text { (inch-pounds) }\end{array}$ & 75,400 & 100,000 \\
\hline
\end{tabular}

The NTF-117S is a monolithic balance, shown in Figure 7. It is made from a single piece of high strength maraging steel. Its overall dimensions are approximately 16 inches in diameter by 25.75 inches long and weighs approximately 900 pounds. It is a five component balance, measuring normal force, axial force, pitching moment, rolling moment, and yawing moment. The balance instrumentation consists of a primary and secondary set of strain gage bridges. Thirty-two platinum resistive temperature detectors (PRTDs) monitor the balance temperature profile. These temperature sensors are strategically located to 
provide a global temperature profile as well as localized measurements near the strain gages. The balance design also contains a two-axis on-board accelerometer that provides an absolute reference of the balance attitude. The on-board accelerometers also provide a direct transfer of the balance calibration coordinate system to the wind tunnel model coordinate system. This is especially important since a flow angularity technique has not been developed for semi-span testing, and therefore the offset between the balance axes and the model axes must be mechanically determined.

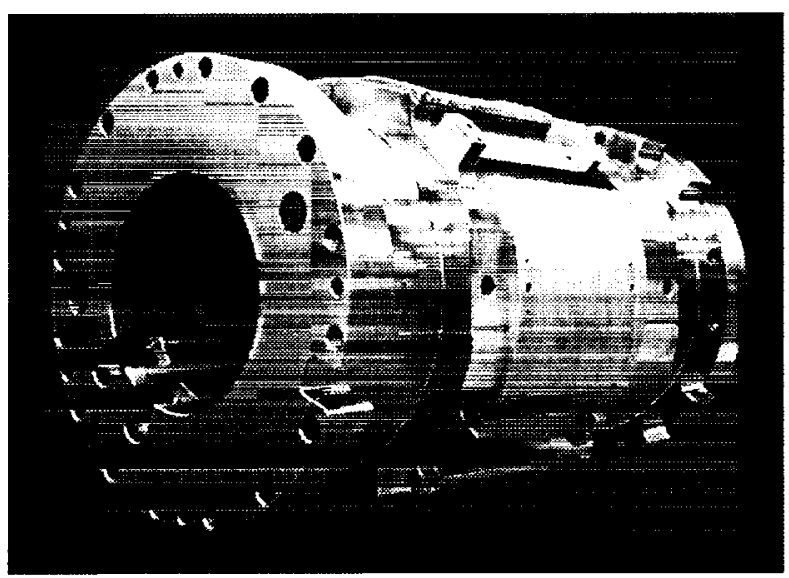

Figure 7. Photograph of balance NTF-I17S.

The design requirements of the NTF-117S were also unique in the area of stiffness. In this case, model dynamics were not the driving factor for the stiffness requirement. The stiffness requirement was due to the unique assembly of the semi-span model system. A detailed discussion of the semi-span system is provided in Reference 13. This assembly requires a minimization of the pitching moment deflection of the metric portion of the model relative to the non-metric standoff. In this system there is a non-contacting seal between the model standoff (non-metric) and the model fuselage (metric). A high torsional (pitching moment) stiffness of the balance was requested to maintain this gap under aerodynamic loading.

During tunnel operations, the balance is heated and maintained at room temperature or above. Since this "hot-balance" concept has been adopted, the calibration of the semi-span balances includes a complete room temperature calibration and primary calibration at 150 degrees Fahrenheit. The elevated temperature calibration is used to determine coefficients that compensate for the change in sensitivity as a function of temperature.
The design and fabrication of this second generation NTF semi-span balance has been completed. This balance will provide enhanced force measurement capability for continued investigations of high-lift systems of aircraft at near flight Reynolds numbers.

\section{Internal NTF Balance Thermal Compensation}

All force balances under go a measure of thermal compensation. The level of thermal compensation is dictated by the facility environmental conditions in which the balance will be used. Due to the extreme temperature and pressure environment at the NTF, temperature compensation of the balance is critical to the overall data quality and productivity. In an integrated view of aerodynamic data quality produced by the NTF, it is clear that the balance thermal compensation is not the only contributing factor, but this discussion is limited to the thermal response of the force balance.

None of the internal balances at the NTF are thermally controlled during the collection of aerodynamic data. A balance temperature conditioning system at the NTF has provided improved productivity in decreasing the time required for the balance to reach the same temperature as the tunnel gas. This conditioning system is not used during data collection due to the possibility of influencing the local flow field around the model, and thereby corrupting the aerodynamic data. Therefore, since the balance temperature is not controlled the fluctuations in the temperature profile of the balance are compensated by various means.

Significant research in the area of thermal compensation has been performed. The following sections will provide an introduction to the balance thermal gradient issues, an overview to the various stages and methods that are currently available for the thermal compensation of internal NTF balances and a brief presentation of research efforts.

The measuring of forces and moments on wind tunnel models without thermally controlled balances is challenging at cryogenic conditions due to the strain gages producing output caused by differential thermal expansion. Any thermal effects on the balance are desired to be considerably less than the balance force measurement accuracy. Current NTF balances can have uncorrected thermal effects that are larger than the balance accuracy under extreme gradient conditions. Therefore, a thermal compensation strategy is required. 
Temperature changes of the balance cause real strain that is indistinguishable to the individual strain gage from an applied external force. That is, varying temperatures on the balance will cause the balance to bend and deform and thus produce a real strain. This strain occurs because of differential expansion (or contraction) of the balance due to the balance structural temperature profile.

Temperature effects can be segmented into two distinct areas, namely temperature changes that result in an isothermal temperature condition on the balance and changes that result in a spacial thermal gradient across the balance. The term isothermal refers to a condition where the balance is soaked at a specific temperature, and therefore no significant thermal gradient exists. During wind tunnel testing, both of these conditions exist in various levels simultaneously.

Solution approaches that are discussed below include those that attempt to isolate and individually compensate for these effects and those that treat the thermal effects in a combined compensation scheme. These approaches to thermal compensation span the full realm of developing an understanding of the underlying physics of the phenomena to an empirical curve fitting approach. Proposed solutions also differ in their implementation. The implementation strategies of these methods include those that perform the compensation by installation of physical sensors on the balance and others are performed as numerical algorithms within the data reduction software.' Ulimately, the thermal compensation strategies used at the NTF include a combination of both of these various approaches.

Temperature influences all of the balance components. Due to the structural design of the NTF balances, thermal gradients primarily affect the axial force measurement section. This high thermal sensitivity of axial force coupled with the aerodynamic importance of this measurement has resulted in a focused effort of full thermal compensation of the axial force component. Presented below will be a review of current thermal compensation practices followed by a presentation of solution approaches under research.

\section{Current Techniques of Thermal Compensation}

Thermal compensation of the balance begins in the design phase with the strategic placement of the individual strain gages on the balance structure. This step focuses on minimizing the magnitude of thermal effects through the use of a Wheatstone bridge electrical configuration and geometric symmetry in the individual gage placement. Following the design, the gage matching and installation procedures, previously discussed, attempt to minimize the magnitude of the thermal effects. This procedure analyzes the individual gage characteristics and selects an optimum combination of four gages. A low thermal expansion coefficient material is used for the gage matching procedure to isolate the gage characteristics from the influence of the balance structural material, Once the balance is fully instrumented and thermally tested, the residual thermal effects are compensated in a two-step process.

First, installing a section of temperature sensitive wire within the proper leg of the strain gage bridge compensates the isothermal temperature effects on the electrical zero of the balance. This compensation only addresses steady state isothermal temperature change, not steady state or transient thermal gradient temperature profiles. After the physical compensation is complete, the residual isothermal temperature response is compensated using a mathematical model. This quadratic math model derives a relationship between the isothermal output of the balance as a function of the local temperature at the bridge location. ${ }^{4}$

After the isothermal compensation is completed, the effects of thermal gradients on the axial force component are compensated. Recall that there are two axial force bridges on the commonly used NTF balances. These bridges provide equivalent sensitivity and are electrically isolated from each other. Two implementations of the same method are applied to these axial force bridges. In the primary axial bridge. four temperature sensors are placed in strategic locations around the axial force measurement section. These four sensors are electrically connected within the primary axial force bridge. The composite temperature response of these sensors compensate for the thermal gradients in the axial force section. They are installed in a manner that attempts to have them read only the temperature of the balance structure and not strain. An iterative approach is used to select the resistance value of the sensors. This method has been used extensively on NTF balances. There are two primary disadvantages to this method. First, the sensors are quite vulnerable to damage and since they are electrically connected within the strain gage bridge this damage can result in a loss of the axial force measurement. Second, the thermal gradient compensation provided by these sensors is optimized for a specific gradient profile obtained in the strain gage laboratory without a wind tunnel model installed. The gradient profile that is observed during 
wind tunnel testing could be quite different. In order to address these reliability and data quality problems, an alternate implementation of the same method was applied to the secondary axial force bridge.

The secondary axial force bridge does not have sensors electrically connected within the bridge. Instead four PRTDs (platinum resistive temperature detectors) are installed in symmetric locations relative to the sensors used in the primary bridge. These PRTDs provide the same form of a composite temperature measurement that can be correlated to the thermal gradient response of the axial force bridge. In this bridge, the compensation is implemented in the software. It requires the derivation of a mathematical model that provides the relationship between the four PRTDs and the axial force response.

Advantages to this implementation include the isolation of the strain gage bridge from the vulnerable external mounting of the temperature measurements performed by the PRTDs. Also, the determination of the coefficients of the math model can be derived from data obtained during a cryogenic excursion that simulates the wind tunnel test environment with the model system fully assembled. This method has been used since 1997, and has been proven to provide an equivalent compensation as the method used in the primary bridge. In fact, this method has demonstrated consistently that inadequate compensation can result unless it is performed with a full model assembly and within the temperature profile that is obtained during the wind tunnel test. To determine this typical temperature profile, a study of wind-on tunnel data was conducted and is discussed in a subsequent section.

After the implementation of the above techniques there remains a residual effect of thermal gradients on the axial force component. The primary wind tunnel strategy for overcoming this residual effect is to obtain multiple wind-off reference readings at the specific test conditions and to set a boundary on the acceptable temperature gradient across the balance. These strategies are effective in minimizing the residual temperature effects and provide adequate data quality, but it would be more desirable from a productivity perspective to not require these multiple wind-off reference readings and the thermal conditioning of the balance. Furthermore, temperature variation inbetween these wind-off references potentially could be better compensated. Therefore, there is ongoing research into obtaining a better understanding of the thermal response phenomena and developing better compensation techniques. An overvicw of these techniques is provided in the next section.

\section{Research into Improved Methods of Thermal Compensation}

In order to improve the thermal performance of force balances, research is ongoing in three main areas. First, a research project has been initiated to better understand the underlying physics of the thermal gradient phenomena. Second, a study has been performed to quantify the operational temperature profiles of a balance during wind tunnel testing. Third, an empirical curve fitting approach has been tested on various model configurations. An overview of these approaches is provided below. The results of these research efforts have broad applications to all force balances, not only those that operate at the NTF.

\section{TIGER: Thermally Induced Gradient Effects Research}

To find a robust solution to thermal compensation, the underlying physics of the phenomena must be understood. This is the premise of the ThermallyInduced Gradient Effects Research (TIGER) project. ${ }^{14}$ The TIGER approach is to generate various steady state and gradient temperature profiles on a speciallyprepared "research" balance. The research balance is instrumented with 118 temperature sensors, 64 strain gages, and 40 foil resistive heater elements. This balance will be subjected to a variety of temperature and load configurations.

In much the same way that a balance undergoes force calibration, the research balance will undergo a temperature calibration. A mathematical model will be developed that relates the electrical response of the balance to the temperatures on the balance structure. In this case, the independent variables are the temperature measurements on the balance structure, which are analogous to the applied loads during a force calibration.

The major difference between this temperature calibration and force calibration is the correlation of the response of the strain gages. In a force calibration the strain gages are located to provide maximum sensitivity to the applied load. This can be done because the relationship of applied load to strain gage response is well understood. The mathematical model is selected based on an understanding of the physics based relationships of strain as a function of applied loads. This model is an approximation to the actual functional relationship, but it is based on the field of structural 
mechanics. In the temperature calibration case, the temperature sensor responses may not be well correlated to the strain gage response and the relationship is not well understood. There are a number of modes of heat transfer that influence the temperatures measured on the balance. If a robust model can be obtained, then it would provide a means to compensate for the thermal response of the balance. It would also provide the balance designer with insight into how the structure of the balance could be modified to reduce the sensitivity to temperature.

A finite element model has been constructed and verified for the research balance. The testing of the research balance at temperature is time consuming and there are many possible temperature profiles that can be generated. It is believed that the finite element model can be used to reduce the amount of experimental lesting required by isolating the main effects of the thermal response.

Another challenge in the TIGER project is how to analyze the data from the research balance. Considering all combinations of temperature sensors leads to more than $3.6 \times 10^{35}$ possible combinations. Clearly, an exhaustive search to find the best combination is not feasible. A variety of nontraditional modeling techniques have been considered. These techniques include: linear regression, neural network, eigenvector decomposition, gradient search strategies and genetic algorithms. At this time only a preliminary set of data has been obtained from the research balance and therefore the results of these techniques have not been thoroughly evaluated.

\section{Balance Operational Temperature Profile Study}

In all compensation schemes, the temperature range that requires compensation must be defined. Recall that the current techniques do not provide a robust compensation scheme for all temperature profile conditions. However, these techniques do perform acceptable compensation over the range in which they are optimized. If the temperature profile of the balance can quantified, then current techniques can be optimized over a specific temperature range and therefore provide better compensation results.

A study has been performed on data collected during three NTF wind tunnel tests. The data has been analyzed in a number of different ways. One of the most challenging tasks in this analysis was the definition of the temperature gradient. The temperature gradient across the balance structure can be described in different ways. For example, the temperature difference from the forward portion of the balance to the rear portion of the balance is one gradient definition. Alternatively, the temperature difference between the forward portion to the middle portion is another gradient definition. There are many possibilities for the definition of the gradient, but the criteria for a good definition is how well it is correlated with the balance electrical response. The results from this study indicate that the best known measure of the gradient is the composite four temperature sensors measurement made locally in the axial force section.

Another more significant result from this study is that the current compensation schemes are performed and evaluated over temperature conditions that are not representative of the operational balance temperature profiles. The current compensation techniques are performed over a significantly larger temperaturc gradient range than is imposed during wind tunnel operations. Few data points are available from thermal testing prior to the wind tunnel entry that are in the appropriate gradient range. The residual thermal response of the balance within this operational range is on the order of the balance accuracy. This further indicates that the temperature profiles seen during wind tunnel testing produces significantly lower thermal response of the balance than has been historically attributed to balance thermal errors.

\section{Empirical Modeling}

Another approach involves the modeling of a thermal excursion performed prior to the wind tunnel entry. This model would then provide a means to compensate the thermal response of the balance during wind tunnel testing. This approach, proposed by Richard Wahls of NASA LaRC, has been evaluated on different model configurations. The proposed model considers the thermal response of the balance as a quadratic relationship to the isothermal temperature (or local average temperature) of the balance and as a linear relationship to the temperature gradient. Stated another way, the balance thermal response is second order in temperature and first order in gradient. The complete mathematical model involves the product of the isothermal function and the gradient function. A regression is performed to determine the six coefficients in the model. This model integrates the isothermal temperature response with the temperature gradient response of the balance. It is the only combined approach that has been proposed. The model is considered to be empirical, but may be found to be physics based in future research. 
This model has been used to perform sensitivity studies that provide insight to the magnitude of thermal response as a function of the tunnel test temperature and the balance temperature gradient profile. This has also been useful in the separation of other thermal errors such as the parasitic loading effects of the tubing and wiring that parallels the balance inside the model.

The above efforts provide insight into an improved compensation scheme for force balance thermal response. These efforts will be beneficial regardless of the strain sensing device installed on the balance or the experimental off-body deflection based techniques that have been proposed for future force measurement systems. Also, regardless of the strain sensors used for the force transducer, the temperature environment will influence the structural members and thereby produce strain that is indistinguishable from externally applied loads. In any case where the aerodynamic loads are found based on the deflection of structural members, the thermal effects on the structure must be adequately compensated.

In general, thermal response of the balance is undesirable. It is challenging to compensate for these thermal effects because they are indistinguishable from externally applied loads. A multi-tiered approach has been applied to thermal compensation that starts with the balance design and encompasses all phases of balance production and wind tunnel operation. A robust compensation scheme is required that can be optimized and tested in the operational temperature profile range that is seen during wind tunnel testing.

\section{NTF Force Balance Calibration}

The primary purpose of calibration is to derive a mathematical model that is used to estimate the aerodynamic loads exerted on the balance during wind tunnel testing. Furthermore, the quality of this model is also determined during the balance calibration. This phase of the balance production also experimentally tests the balance structure and the strain gage installation.

In general, force balance calibration consists of setting independent variables and measuring the response of the dependent variables. The applied loads are the independent variables and the electrical responses of the balance are the dependent variables. Current math models are based on a quadratic polynomial. The range of load set in the calibration experiment defines what is referred to as the inference space. The error of estimation of the math model is evaluated within this space.

Current force balance calibration schedules increment one independent variable at a time. Each independent variable is incremented throughout its full-scale range. During this incrementing of the primary variable, all other variables are zero, or are held at a constant magnitude. This approach is referred to as one-factorat-a-time (OFAT) experimentation.

All of the required set points of independent variables are grouped into a load schedule, or calibration design. Ordering of the points within the design is based on the efficiency of the load application system and specific data analysis algorithms. For optimum design execution, efficiency is gained by the application of one load at a time in incremental levels. Data analysis algorithms used to determine the balance calibration matrix often rely on a specific grouping of independent variable combinations. Single variable loading is used to calculate the main linear and quadratic effects. Constant auxiliary loads, in combination with single incremented loads, are performed in order to assess two factor interaction effects. A typical calibration design, used to derive and evaluate a quadratic model, contains approximately 900 data points.

\section{Current Calibration Methodology}

The NASA LaRC manual calibration consists of applying dead weights with precision-machined hardware, state-of-the-art leveling devices, and data acquisition instrumentation to determine all first and second order interactions. ${ }^{7}$ After each loading, the balance is re-leveled prior to taking data to assure that the applied loads are orthogonal with the balance coordinate system. This type of calibration system is often referred to as a repositioning system. There are a total of 82 load sequences performed to calculate the calibration mathematical model coefficients. Each load sequence consists of four ascending increments and four descending increments providing a total of 738 data points. Also, three sets of multi-component proofloading are performed to assess the quality of the math model. As previously described, a complete calibration is performed at room temperature and single component loading is performed at cryogenic temperature.

Manual dead weight balance calibration stands have been in use at LaRC since the $1940^{\circ} \mathrm{s}$. They have undergone continuous improvement in load application, leveling, and data acquisition. These manual systems are the backbone or "standard" that other balance 
calibration systems have been compared against. The simple accurate methodologies produce high confidence results.

There are two primary disadvantages to the current manual calibration system. First, the time required performing a manual calibration makes it prohibitive to provide quick turnaround response to the wind tunnels as well as perform repeated calibrations for statistical analyses. Second, the inability to perform complete calibrations at environmental conditions in which the balance will operate in the wind tunnel. The primary environmental parameters are temperature and pressure. $\mathrm{LaRC}$ is involved in various research and development efforts to address these productivity and data quality limitations. An overview of three of these research projects is presented in the following sections.

\section{Automatic Balance Calibration Systems}

To address the requirements of faster calibrations semi/automatic systems have been investigated. The concept of automatically calibrating a strain-gage balance has been considered at NASA LaRC for more than 40 years. LaRC used semi-automatic machines from the 1950's to the 1980's as a primary means of balance calibration. The effort required maintaining critical alignment as well as the aging control systems of the semi-automatic systems rendered them obsolete in the late 1980's. At that time, fully automated balance calibration systems (ABCS) were beginning to be developed around the world. LaRC has evaluated these systems. ${ }^{15,16}$

The primary benefit of an automated calibration system is the increased speed of calibration. In general, all automatic balance calibration systems are designed to simulate the manual calibration process. Unfortunately, the automation of this tedious manual process results in a complex system. The mechanical complexity of an automated system makes them expensive to manufacture and difficult to determine their load application accuracy. Automated balance calibration systems can reduce calibration time from three to four weeks to a few days.

An evaluation process was performed using a LaRC state-of-the-art cryogenic force balance (Balance NTF$113 \mathrm{~B}) .^{17}$ This balance was calibrated in two different automated systems and were compared to the results of the LaRC manual calibration system. This study was limited to a comparison of room temperature calibration results. The significant conclusion of this study was that a mixture of an abbreviated manual calibration and complete environmentally simulated automatic calibration would be required in standard practice. The manual calibration, in its simplicity, inherently provides more confidence in the data collected. An automatic balance calibration system that is able to thermally condition and calibrate the NASA LaRC NTF-113 class of balances to their full-scale load limits is currently not available anywhere in the world.

Even though the automated systems provide a significant reduction in calibration time, the quality of the calibration is not equivalent to the manual dead weight system. Furthermore, there does not exist an adequate ratio of the calibration system accuracy to the balance undergoing calibration, and therefore it is not possible to achieve the attainable accuracy for state-ofthe-art force transducers. Improvements to automated systems will be challenging due to their mechanical complexity.

\section{Variable Temperature Calibration Stand}

High-precision calibration of force balance at clevated or cryogenic temperature has been addressed with the construction of a new facility at LaRC. This facility features a variable temperature calibration system that enables the balance to be thermally conditioned within the range of $-250^{\circ} \mathrm{F}$ to $+150^{\circ} \mathrm{F}$. The balance and all associated calibration hardware are contained in a thermal chamber. The convection-based chamber is non-contacting with the metric portion of the balance. This non-contact relationship eliminates parallel load paths across the balance that causes parasitic loads. Load application is based on manual dead weight loading. A simplified drawing of the system is provided in Figure 8. The system is in the process of becoming operational.

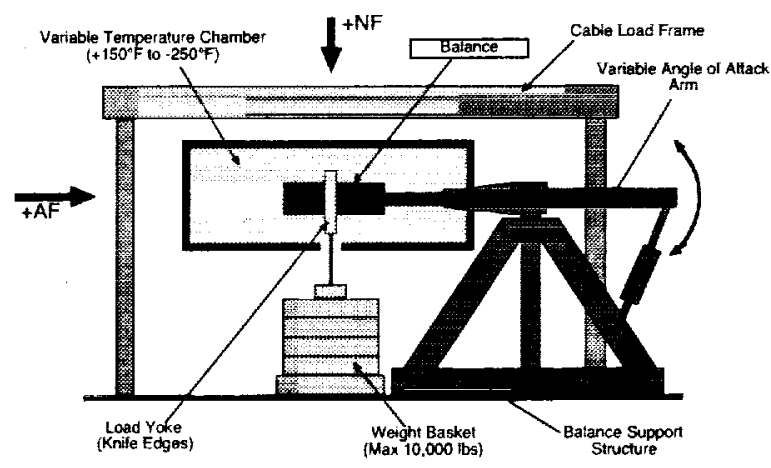

(a) Simplified drawing.

Figure 8. Variable Temperature Calibration Stand. 


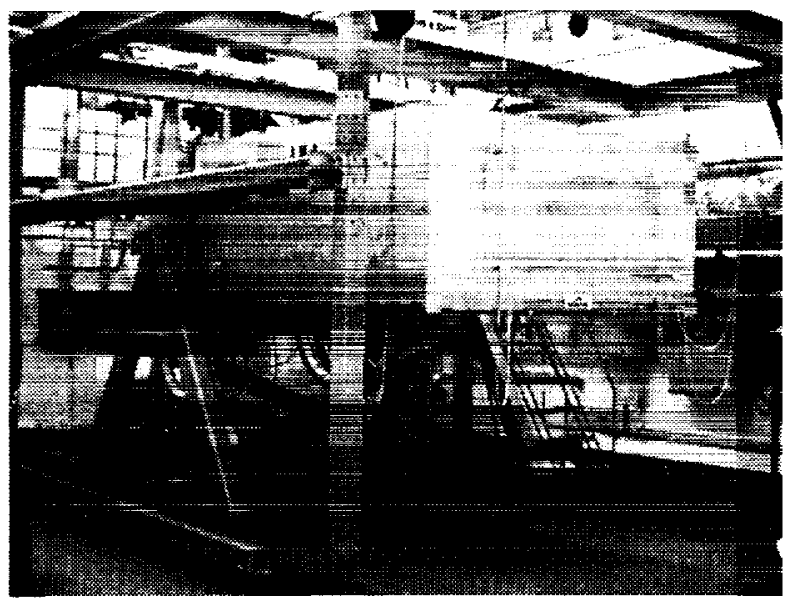

(b) Photograph of installed system.

Figure 8. concluded.

Once commissioned, this system will provide LaRC with the capability to perform calibrations at elevated or cryogenic temperatures. The system is capable of full calibrations, but it would require months of calibration time to perform a full calibration. Therefore, full calibrations at temperature will be limited to research efforts not production calibration. The production use of the system will include the capability of determining non-linear corrections for component sensitivity shifts as a function of temperature. Current sensitivity corrections are limited to a linear coefficient due to the limitation of the current two-temperature level calibration procedure discussed previously. The ability to achieve intermediate temperatures will enable LaRC to more fully characterize the sensitivity corrections using a non-linear function.

This system address the data quality limitations related to environmental calibrations, but will lengthen the calibration time. The initial use of the system will be to quantify the benefits of performing variable temperature calibrations. It will also determine the minimum number of calibration loads to be performed at temperature to adequately compensate for thermal effects. The trade-off of increased calibration time versus improved data quality will need to be assessed.

\section{Modern Design of Experiments Applied to Balance Calibration}

LaRC has been conducting research in a "modern design of experiments" (MDOE) approach to force balance calibration. Formal experimental design techniques provide an integrated view to the force balance calibration process. This scientific approach applies to all the three major aspects of an experiment: the design of the experiment, the execution of the experiment, and the statistical analysis of the data. A loading schedule based on MDOE will be subsequently referred to as a calibration design.

Formal experimental design has been applied to wind tunnel testing at NASA LaRC since January 1997. ${ }^{18}$ The application of formal experiment design to balance calibration was first considered in 1980 at NASA LaRC. ${ }^{19}$ A classical quadratic experimental design was conducted in 1980, and the results appeared promising. Due to the complex load application requirements of the MDOE design, this effort was considered not to be feasible for production calibration, and the idea remained dormant for nearly two decades. In 1999, a new approach to force balance calibration was considered, and a custom MDOE design was developed. ${ }^{20}$

A MDOE approach deviates from the current trend of collecting massive data volume, specifying ample data to meet requirements quantified in the design but without prescribing volumes of data far in excess of this minimum. The goal is to efficiently achieve the primary objective of the calibration experiment; namely the determination of an accurate mathematical model to calculate the unknown loads from measured balance responses. MDOE also provides a systematic approach for the investigation of higher order mathematical models.

A new system of hardware has been developed that is integrated with a custom developed MDOE calibration design, see Figure 9. This system enables the complete calibration of a six component force balance with a single force vector. The system manipulates the balance in three-dimensional space, while keeping the calibration load aligned. The use of a single vector calibration load reduces the set-up time for the multiaxis load combinations needed to generate a complete calibration mathematical model. The system also reduces load application inaccuracies caused by the conventional requirement to generate multiple force vectors.

The integrated hardware system and calibration design optimizes the calibration process. The simplicity of the system coupled with nearly an order of magnitude reduction in data volume reduces calibration time from three to four weeks to approximately three days. The advantage to this system is that it improves on the "trusted" aspects of current manual calibration system, and therefore there is a high confidence in the results. The single-vector balance calibration system improves the data quality, while simultaneously improving 
productivity. The system is designed to integrate a thermal conditioning system and will provide the additional capabilities of environmental calibration.

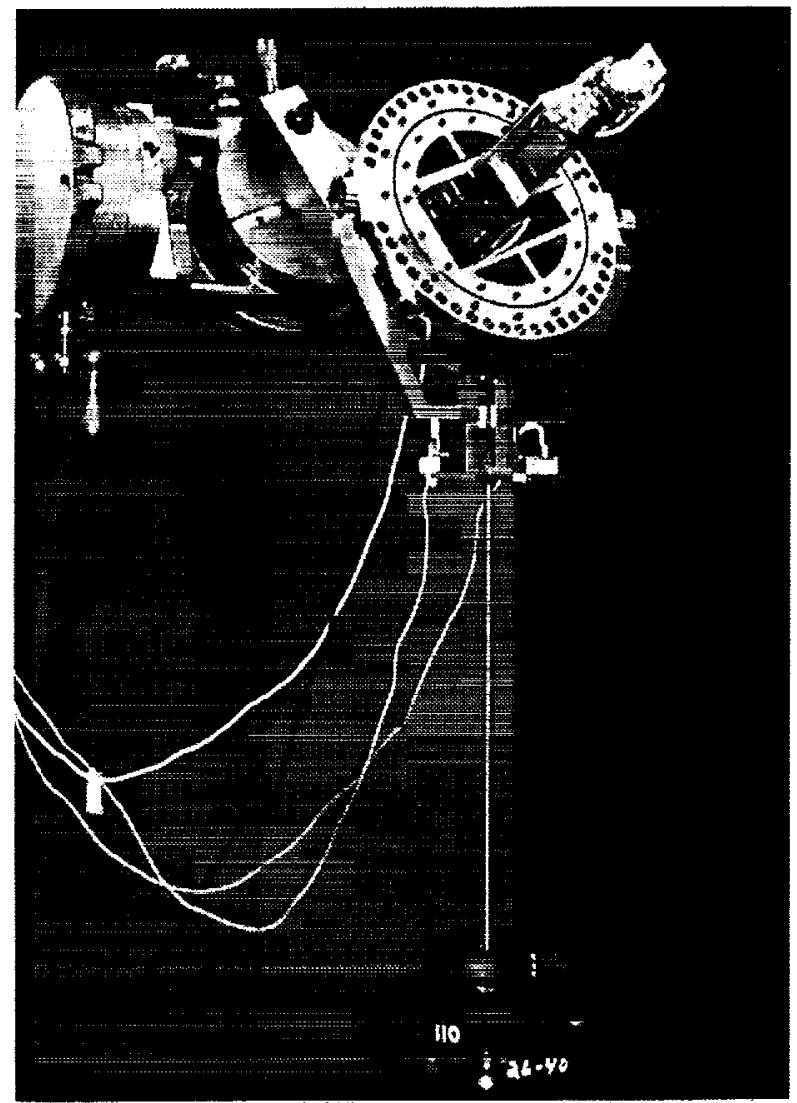

Figure 9. Photograph of the Single-Vector Force Balance Calibration System.

\section{Areas of Future Research}

Areas of future research are judged to be in balance fatigue and fracture analysis, alternative balance materials, balance to model mechanical connections, improved fabrication methods, strain sensor technology, refinement of dynamic design requirements, and the integration of the model attitude measurement with the force balance.

Currently, balance operational life is not considered to be finite in terms of fatigue and fracture. Furthermore, there is no mechanism in place that automatically records the dynamic history on all balances. Recent advancements in fatigue and fracture analysis techniques need to be applied to force balances. ${ }^{21}$ These techniques will rely on either the assumptions about the dynamic loading of a balance in the wind tunnel or recording the actual balance load history. The implementation of these analysis techniques could have a significant impact to the operational life of the balance.

Alternative materials that can be used for balance fabrication could provide desired improvements in stiffness, cryogenic toughness, and higher strength, thereby providing increased safety margins. Studies are ongoing in the area of alternative materials for cryogenic model fabrication. ${ }^{21}$ This will provide valuable information for the balance designer. Additional criteria for force balances must be considered, such as low material hysteresis and intricate machining requirements.

Balance accuracy is greatly influenced, and many times limited, by the quality of the mechanical connections to the model system. These connections, which are in close proximity to the sensitive strain measurement sections, can introduce hysteresis into the force measurements. Developments in this area can simultaneously improve data quality and reduce model system installation time. A novel application of a Marman clamp attachment was considered for the NTF$116 \mathrm{~A}$, but was not implemented due to inadequate time to perform analysis and testing. Other systems of attachment should also be considered and evaluated based on data quality, manufacturing cost, and ease of tunnel implementation.

Improved fabrication methods are being studied. The primary goal of this effort is reducing the time and cost of the production of force balances. Fabrication techniques that are being evaluated involve state-of-theart conventional and electro-discharge capabilities. Also, non-traditional techniques that remove the restrictions imposed by the fabrication of the balance from a single piece of material may provide the balance designer with more freedom in the force transducer design.

Improvements in strain sensor technology are required to increase force measurcment resolution. Strain measurement using conventional foil resistive strain gages manufactured from other alloys, such as platinum, can increase the gage factor by two and thereby double measurement resolution. Also, fiberoptic strain sensors have the potential to increase resolution. ${ }^{22}$ The implementation of fiber-optic strain sensors may require the structural re-design of the balance measurement sections.

The development of the NTF-116A, previously discussed, integrated static and dynamic design criteria. The design demonstrates that the balance measurement 
sensitivity can be de-coupled from the overall stiffness. The primary obstacle to the broad application of this technique to suppress model vibrations is the definition of dynamic design requirements. These requirements most likely will be facility dependent and possibly model dependent. Also, advanced model support systems must be integrated into the concept of model system de-tuning.

Aerodynamic performance coefficients that are obtained during wind tunnel testing are a function of the wind tunnel environment and numerous instruments. The two primary instruments are force balance and the angle-of-attack (AOA) device that measures model attitude. These instruments are individually produced and calibrated, and their inclusion into the model design is considered independently. Previous model designs have included sub-optimum mechanical alignment of the AoA axes with the balance axes. Furthermore, these connections often lack the rigidity to ensure that the alignment is maintained under aerodynamic loads. An integrated measurement device that includes force and attitude measurement has been considered. This device would not only improve combined measurement data quality but would also minimize the volume required inside the model for the independent mounting of these two instruments. This combined measurement device would be calibrated as a system and not rely on the mechanical alignment of the axes within the model.

\section{Concluding Remarks}

An introduction to the unique requirements that are posed by the NTF has been provided. An overview of current force measurement capabilities at the NTF has been presented. The requirement for a comprehensive view of the balance design, production, calibration, maintenance, and use in the wind tunnel has been discussed. The involvement of the force balance engineer into the NTF wind tunnel measurement environment has been emphasized. New balance development projects have been described that will extend the NTF force measurement capabilities. Research into improved thermal compensation and balance calibration has been presented.

It is clear that there are many opportunities for advancing the state-of-the-art in force balance technology based on the areas of future research that have been presented. These advancements will improve the effectiveness of the wind tunnel testing cycle in producing a better understanding of aircraft performance.
The force measurement requirements at the NTF are challenging due to the harsh environment and the emphasis on data quality. LaRC continues to fulfill these requirements and is constantly developing new and innovative methods of improving them.

\section{Acknowledgements}

The author would like to thank Judy Ferris, retired NASA LaRC research scientist, for her years of foundational development activities in the area of cryogenic balance technology, and Tom Moore, of the Model Systems Branch at LaRC, for his expertise in the development of many of the strain gage installation techniques used on NTF balances. Also, Mark Roth of Modern Machine and Tool Company for his expertise in strain gage installation, moisture resistant coating application and periodic review of the NTF force balances.

\section{References}

1) Baals, D.D.; Corliss, W.R.: Wind Tunnels of NASA. NASA SP-440, 1981.

2) Ferris, A.T.: Force Instrumentation for Cryogenic Wind Tunnels Using One-Piece Strain-Gage Balances. NASA TM 81845, 1980.

3) Rhew, Ray D.: NASA LaRC Strain Gage Balance Design Concepts. Paper DE-5, First International Symposium on Strain Gauge Balances. October 1996.

4) Foster, J.M.; Adcock, J.B.: User's Guide for the National Transonic Facility Research Data System. NASA TM-1 10242, April 1996.

5) Ferris, A.T.: Cryogenic Strain Gage Techniques used in Force Balance Design for the National Transonic Facility. NASA TM 87712, 1986.

6) Moore, T.C., Sr.: Strain Gages in Use at NASA Langley - A Technical Review. Paper SG-2, First International Symposium on Strain Gauge Balances. October, 1996.

7) Ferris, A. T.: Strain Gauge Balance Calibration and Data Reduction at NASA Langley Research Center. Paper DR-2, First International Symposium on Strain Gauge Balances. October, 1996.

8) Lindell, M..: Finite Element Analysis of a NASA National Transonic Facility Wind Tunnel Balance. Paper FE-1, First International Symposium on Strain Gauge Balances. October 1996. 
9) Buehrle, R.D.: System Dynamic Analysis of a Wind Tunnel Model with Applications to Improve Aerodynamic Data Quality. University of Cincinnati, Division of Research and Advanced Studies, Dissertation 1997.

10) Parker, P.A.: Wind Tunnel Model System Dynamic Analysis and Simulations with Application to Model System Vibration Suppression. Christopher Newport University, Department of Applied Physics and Computer Science, Thesis, 2000.

11) Young, Clarence P.: Model Dynamics. AGARD R812, February 1997.

12) Edwards, R.W.: An Approach for Increasing Aeroelastic Divergence Dynamic Pressure of Wind-Tunnel Models. AIAA Paper No. 92-4002, July 1992.

13) Gatlin, G.M., Parker, P.A., Owens,L.R.,Jr.: Development of a Semi-Span Test Capability at the National Transonic Facility. AIAA Paper No. 2001-0760, 39 ${ }^{\text {th }}$ Aerospace Sciences Meeting and Exhibit, Reno, NV, Jan. 2001.

14) Hereford, J.M., Parker, P.A., Rhew, R.D.: TIGER: Development of Thermal Gradient Compensation Algorithms and Techniques. Second International Symposium on Strain Gauge Balances, May 1999.

15) Levkovitch, Michael: Automatic Balance Calibrator at Israel Aircraft Industries. January 1993.

16) Polansky, L.; and Kutney, J. T. Sr.: A New Working Automatic Calibration Machine For Wind Tunnel Internal Force Balances. AIAA 93-2467. June 1993.

17) Parker, P.A.; and Rhew R.D.: A Study of Automatic Balance Calibration System Capabilities. Second International Symposium on Strain Gauge Balances, May 1999.

18) DeLoach, R.: Applications of Modern Experiment Design to Wind Tunnel Testing at NASA Langley Research Center. AIAA 98-0713, 36 ${ }^{\text {th }}$ Aerospace Sciences Meeting and Exhibit, Reno, NV, Jan. 1998.

19) Dahiya, R.C.: Statistical Design for an Efficient Calibration of a Six Component Strain Gage Balance. NASA LaRC Contractor Report, NAS 115648, August 1980.
20) Parker, P.A.; Morton, M.; Draper, N.; Line, W.: A Single-Vector Force Calibration Method Featuring the Modern Design of Experiments. AIAA 2001$0170,38^{\text {th }}$ Aerospace Sciences Meeting and Exhibit, Reno, NV, Jan. 2001.

21) Kimmel, W.M.: Cryogenic Model Materials. AIAA $2001-0757,38^{\text {th }}$ Aerospace Sciences Mecting and Exhibit, Reno, NV, Jan. 2001.

22) Hare, D.A.; Moore, T.C., Sr.: Characteristics of Extrinsic Fabry-Perot Interferometric (EFPI) Fiber-Optic Strain Gages. NASA LaRC TP-2000210639 , December 2000. 